Probl. Anal. Issues Anal. Vol. 6 (24), No. 1, 2017, pp. 19-40

DOI: $10.15393 /$ j3.art.2017.3810

UDC 517.983

V. M. BRUK

\title{
BOUNDARY VALUE PROBLEMS FOR INTEGRAL EQUATIONS WITH OPERATOR MEASURES
}

\begin{abstract}
We consider integral equations with operator measures on a segment in the infinite-dimensional case. These measures are defined on Borel sets of the segment and take values in the set of linear bounded operators acting in a separable Hilbert space. We prove that these equations have unique solutions and we construct a family of evolution operators. We apply the obtained results to the study of linear relations generated by an integral equation and boundary conditions. In terms of boundary values, we obtain necessary and sufficient conditions under which these relations $T$ possess the properties: $T$ is a closed relation; $T$ is an invertible relation; the kernel of $T$ is finite-dimensional; the range of $T$ is closed; $T$ is a continuously invertible relation and others. We give examples to illustrate the obtained results.
\end{abstract}

Key words: Hilbert space, integral equation, boundary value problem, operator measure, linear relation

2010 Mathematical Subject Classification: 46G12, 45N05, $47 A 06$

1. Introduction. In this work we consider the following integral equation on a segment $[a, b]$ :

$$
y(t)=\int_{t_{0}}^{t}(d \mathbf{p}) y(\xi)+g(t)
$$

where $\int_{t_{0}}^{t}$ stands for $\int_{\left[t_{0} t\right)}$ if $t_{0}<t$; for $-\int_{\left[t_{0} t\right)}$ if $t_{0}>t$; and for 0 if $t_{0}=t$. Here $\mathbf{p}$ is an operator-valued measure defined on Borel sets $\Delta \subset[a, b]$ and taking 
values in the set of linear bounded operators acting in a separable Hilbert space $H$. We assume that $\mathbf{p}$ is a measure with bounded variation and we extend the measure $\mathbf{p}$ to a segment $\left[a, b_{0}\right] \supset\left[a, b_{0}\right) \supset[a, b]$, letting $\mathbf{p}(\Delta)=0$ for each Borel sets $\Delta \subset\left[a, b_{0}\right] \backslash[a, b]$ (we shall extend other measures in the same way). In (1), $y$ is an unknown function; $g \in \widetilde{C}\left[a, b_{0}\right] ; \widetilde{C}\left[a, b_{0}\right]$ is a set of functions ranging over $H$, bounded on $\left[a, b_{0}\right]$, continuous from the left (in the strong sense) on $\left(a, b_{0}\right]$, and constant on $\left(b, b_{0}\right] ; a \leqslant t_{0} \leqslant b_{0}$. We prove the following statement to obtain subsequent results of this paper.

Theorem 1. For any function $g \in \widetilde{C}\left[a, b_{0}\right]$ there exists a unique solution of equation (1) belonging to the space $\widetilde{C}\left[t_{0}-\delta, b_{0}\right]$, where $\delta=\delta\left(t_{0}\right)>0$ is small enough if $t_{0}>a$ and $\delta=0$ if $t_{0}=a$.

We consider the following equation

$$
y_{s}(t)=c+\int_{s}^{t} d \mathbf{p}(\tau) y_{s}(\tau), \quad c \in H, \quad s \in\left[a, b_{0}\right],
$$

to construct a family of evolution operators, where $c \in H, s \in\left[a, b_{0}\right]$, $s-\delta(s) \leqslant t \leqslant b_{0}, \delta(s)>0$ if $s>a$ and $\delta(s)=0$ if $s=a$. Let $U(t, s)$ be the operator taking each element $c \in H$ to the value of the solution $y_{s}(t)$ of equation (2). We study the properties of the family of operators $U(t, s)$ and, in particular, prove that 1) $U(s, s)=E$ ( $E$ is the identical operator); 2) $U(t, \tau) U(\tau, s)=U(t, s)$ for $s \leqslant \tau \leqslant t$; 3) the function $t \rightarrow U(t, s)$ is continuous from the left with respect to the uniform operator topology.

Using the properties of the family of operators $U(t, s)$, we obtain the following statement.

Theorem 2. Suppose $\mathbf{p}, \mathbf{q}$ are operator measures with bounded variations and $\mathbf{p}, \mathbf{q}$ have no common atoms consisting of one point, i.e., on the segment $\left[a, b_{0}\right]$ there are no singleton sets $\{\tau\}$ such that the inequalities $\mathbf{q}(\{\tau\}) \neq 0, \mathbf{p}(\{\tau\}) \neq 0$ hold together. Let a function $f$ be integrable with respect to the measure $\mathbf{q}$. Then the solution of the equation

$$
y(t)=c+\int_{a}^{t}(d \mathbf{p}) y(s)+\int_{a}^{t}(d \mathbf{q}) f(s), \quad c \in H,
$$

has the form $y(t)=U(t, a) c+\int_{a}^{t} U(t, s) d \mathbf{q}(s) f(s)$. 
We use the obtained results to study the linear relations generated by the equation

$$
y(t)=c+\int_{a}^{t}(d \mathbf{p}) y(s)+\int_{a}^{t}(d \mathbf{m}) f(s),
$$

where $c \in H ; \mathbf{m}$ is a non-negative operator-valued measure with the bounded variation; $f \in \mathfrak{H}=L_{2}(H, d \mathbf{m} ; a, b)$ ( $\mathfrak{H}$ is defined below). We assume that $\mathbf{m}, \mathbf{p}$ have no common atoms consisting of one point.

In general, equation (3) together with boundary conditions generates not linear operators but linear relations (multi-valued operators). We define a maximal relation $L$ as a closure of a set of ordered pairs $\{y, f\}$ such that equality (3) holds. When studying restrictions of the maximal relation, one often needs to establish boundary conditions which generate restrictions with some given properties. In this paper we consider properties (called states) from works [1, 2]. Among these properties there are the invertibility, the continuous invertibility, the Fredholm property, and others. We obtain necessary and sufficient conditions under which the boundary conditions determine restrictions with specified properties.

We note that if the measures $\mathbf{p}, \mathbf{m}$ are absolutely continuous (i.e., $\mathbf{p}(\Delta)=\int_{\Delta} p(t) d t, \mathbf{m}(\Delta)=\int_{\Delta} m(t) d t$ for all Borel sets $\Delta \subset[a, b]$, where the functions $\|p(t)\|,\|m(t)\|$ belong to $\left.L_{1}(a, b)\right)$, then integral equation $(3)$ is transformed into a differential equation with a weight function. Linear relations and operators generated by such differential equations were considered in many works (see [5], 9], further detailed bibliography can be found, for example, in [3]). We also note that linear relations were first employed in work [10] for the description of extensions of differential operators in terms of boundary conditions.

2. Solutions of integral equations. Let $H$ be a separable Hilbert space with the scalar product $(\cdot, \cdot)$ and the norm $\|\cdot\|$. We consider a function $\Delta \rightarrow \mathbf{P}(\Delta)$ defined on Borel sets $\Delta \subset[a, b]$ and taking values in the set of bounded linear operators acting in $H$. The function $\mathbf{P}$ is called an operator measure on $[a, b]$ (see, for example, [4, ch. 5]) if it is zero on the empty set and the equality $\mathbf{P}\left(\bigcup_{n=1}^{\infty} \Delta_{n}\right)=\sum_{n=1}^{\infty} \mathbf{P}\left(\Delta_{n}\right)$ holds for disjoint Borel sets $\Delta_{n}$, where the series converges weakly. Further, we extend to a segment $\left[a, b_{0}\right] \supset\left[a, b_{0}\right) \supset[a, b]$ any measure $\mathbf{P}$ on $[a, b]$, letting $\mathbf{P}(\Delta)=0$ for each Borel sets $\Delta \subset\left[a, b_{0}\right] \backslash[a, b]$. 
By $\mathbf{V}_{\Delta}(\mathbf{P})$ we denote $\mathbf{V}_{\Delta}(\mathbf{P})=\rho(\Delta)=\sup \sum_{i}\left\|\mathbf{P}\left(\Delta_{i}\right)\right\|$, where sup is taken over finite sums of disjoint Borel sets $\Delta_{i} \subset \Delta$. The number $\mathbf{V}_{\Delta}(\mathbf{P})$ is called the variation of measure $\mathbf{P}$ on the Borel set $\Delta$. Suppose that the measure $\mathbf{P}$ has the bounded variation on $[a, b]$. Then for $\rho$-almost all $\xi \in[a, b]$ there exists an operator function $\xi \rightarrow \Psi_{\mathbf{P}}(\xi)$ such that $\Psi_{\mathbf{P}}$ possesses the values in the set of bounded linear operators acting in $H$, $\left\|\Psi_{\mathbf{P}}(\xi)\right\|=1$, and the equality

$$
\mathbf{P}(\Delta)=\int_{\Delta} \Psi_{\mathbf{P}}(\xi) d \rho
$$

holds for each Borel set $\Delta \subset[a, b]$. The function $\Psi_{\mathbf{P}}$ is uniquely determined up to values on a set of zero $\rho$-measure. The integral sums for (4) converge with respect to the usual operator norm (4, Ch. 5]). Obviously, $\mathbf{V}_{[a, b]}(\mathbf{P})=\mathbf{V}_{\left[a, b_{0}\right]}(\mathbf{P})=\rho([a, b])$.

The function $h$ is integrable with respect to the measure $\mathbf{P}$ on a set $\Delta$ if there exists the Bochner integral in the left-hand side of equality

$$
\int_{\Delta} \Psi_{\mathbf{P}}(t) h(t) d \rho=\int_{\Delta}(d \mathbf{P}) h(t) .
$$

It follows from (5) that if a Borel measurable function $h$ is bounded, then

$$
\left\|\int_{\Delta}(d \mathbf{P}) h(t)\right\| \leqslant \sup _{t \in \Delta}\|h(t)\| \rho(\Delta) .
$$

Let a function $h$ be integrable with respect to the measure $\mathbf{P}$ on $\left[a, b_{0}\right]$. Then the function $y(t)=\int_{t_{0}}^{t}(d \mathbf{P}) h(s)$ is continuous from the left.

Suppose a segment $\left[l_{1}, l_{2}\right] \subset\left[a, b_{0}\right]$. We consider a set of functions ranging in $H$, bounded on $\left[l_{1}, l_{2}\right]$, continuous from the left (in the strong sense) on $\left(l_{1}, l_{2}\right]$, and constant on $\left[l_{1}, l_{2}\right] \cap\left(b, b_{0}\right]$. We introduce the norm $\|u\|_{\left[l_{1}, l_{2}\right]}=\sup _{t \in\left[l_{1}, l_{2}\right]}\|u(t)\|$ on this set and obtain a Banach space denoted by $\widetilde{C}\left[l_{1}, l_{2}\right]$ (or by $\widetilde{C}(\mathcal{I})$, where $\mathcal{I}$ is a segment).

We consider the equation (1), where the measure $\mathbf{p}$ has the bounded variation on $[a, b], a \leqslant t_{0} \leqslant b_{0}, g \in \widetilde{C}\left[a, b_{0}\right]$. We prove Theorem 1 (see the Introduction). 
Proof of Theorem 1. First we shall show that there exists a segment $\mathcal{I}_{\delta, t_{0}}=\left[t_{0}-\delta, t_{0}+\delta\right]$ such that equation (1) has a unique solution in the space $\widetilde{C}\left(\mathcal{I}_{\delta, t_{0}}\right)(\delta>0)$. (We set $\mathcal{I}_{\delta, t_{0}}=[a, a+\delta]$ if $t_{0}=a$ and we set $\mathcal{I}_{\delta, t_{0}}=\left[b_{0}-\delta, b_{0}\right]$ if $t_{0}=b_{0}$.)

Equality (4) holds for the measure $\mathbf{p}$, where $\mathbf{P}, \Psi_{\mathbf{P}}$ are replaced by $\mathbf{p}, \Psi_{\mathbf{p}}$. Let $t \rightarrow \widehat{\rho}(t)$ be a continuous from the left function generating the measure $\rho$. By $\widehat{\rho}_{t_{0}}$ denote the jump of the function $\widehat{\rho}$ at the point $t_{0}$ (it is possible that $\left.\widehat{\rho}_{t_{0}}=0\right)$. We set $\widehat{r}_{t_{0}}(t)=0$ for $t \leqslant t_{0}$ and $\widehat{r}_{t_{0}}(t)=\widehat{\rho}_{t_{0}}$ for $t>t_{0}$. We denote $\widehat{r}\left(t, t_{0}\right)=\widehat{\rho}(t)-\widehat{r}_{t_{0}}(t)$. The function $t \rightarrow \widehat{r}\left(t, t_{0}\right)$ is continuous at $t_{0}$. We introduce the operator measures

$$
\mathbf{r}\left(\Delta, t_{0}\right)=\int_{\Delta} \Psi_{\mathbf{p}}(\xi) d \widehat{r}\left(\xi, t_{0}\right), \quad \mathbf{r}_{t_{0}}(\Delta)=\int_{\Delta} \Psi_{\mathbf{p}}(\xi) d \widehat{r}_{t_{0}}(\xi) .
$$

Then we obtain $\mathbf{p}(\Delta)=\mathbf{r}\left(\Delta, t_{0}\right)+\mathbf{r}_{t_{0}}(\Delta)$.

Under this notation, equation (1) has the form $y=A y+z$, where

$$
\begin{gathered}
(A y)(t)=\int_{t_{0}}^{t} d \mathbf{r}\left(\xi, t_{0}\right) y(\xi)=\int_{t_{0}}^{t} \Psi_{\mathbf{p}}(\xi) y(\xi) d \widehat{r}\left(\xi, t_{0}\right), \\
z(t)=\int_{t_{0}}^{t} d \mathbf{r}_{t_{0}}(\xi) y(\xi)+g(t)=\widetilde{\mathbf{r}}_{t_{0}}(t) x_{0}+g(t), \quad x_{0}=g\left(t_{0}\right),
\end{gathered}
$$

and $\widetilde{\mathbf{r}}_{t_{0}}(t)=0$ for $t \leqslant t_{0}$ and $\widetilde{\mathbf{r}}_{t_{0}}(t)=\mathbf{r}_{t_{0}}\left(\left\{t_{0}\right\}\right)=\mathbf{p}\left(\left\{t_{0}\right\}\right)$ for $t>t_{0}$. Using (6), (7), and the continuity of the function $\widehat{r}\left(\cdot, t_{0}\right)$ at $t_{0}$, we obtain

$$
\|(A y)(t)\| \leqslant \sup _{t \in \mathcal{I}_{\delta, t_{0}}}\|y(t)\|\left|\widehat{r}\left(t, t_{0}\right)-\widehat{r}\left(t_{0}, t_{0}\right)\right|<\varepsilon \sup _{t \in \mathcal{I}_{\delta, t_{0}}}\|y(t)\| .
$$

Consequently, $\sup _{t \in \mathcal{I}_{\delta, t_{0}}}\|(A y)(t)\| \leqslant \varepsilon \sup _{t \in \mathcal{I}_{\delta, t_{0}}}\|y(t)\|$. Using the continuity of $\widehat{r}\left(\cdot, t_{0}\right)$, we take $\delta>0$ such that

$$
\left|\widehat{r}\left(t, t_{0}\right)-\widehat{r}\left(t_{0}, t_{0}\right)\right|<\varepsilon<1
$$

for all $t \in \mathcal{I}_{\delta, t_{0}}$. Then $\|A\|_{\widetilde{C}\left(\mathcal{I}_{\delta, t_{0}}\right)}<1$. Hence the operator $E-A$ has the bounded everywhere defined inverse operator in the space $\widetilde{C}\left(\mathcal{I}_{\delta, t_{0}}\right)$. The function $z$ is equal to zero for all $t$ if and only if $g=0$ on $\mathcal{I}_{\delta, t_{0}}$ (consequently, $x_{0}=0$ ). Hence there exists a unique solution of equation (1) on the interval $\mathcal{I}_{\delta, t_{0}}$. This solution is found by the formula 


$$
y=(E-A)^{-1} z
$$

Now we shall show that there exists a unique solution of equation (1) on the segment $\left[t_{0}-\delta\left(t_{0}\right), b_{0}\right]$. It can be assumed that $t_{0}<b_{0}$. It suffices to prove that if a solution $u$ is defined on an interval $\left[t_{0}-\delta\left(t_{0}\right), d\right)$, where $d<b_{0}$, then $u$ can be extended to an interval $\left[t_{0}-\delta\left(t_{0}\right), d_{1}\right)$, where $d_{1}>d$.

We retain the notation of the above proof, however, we replace $t_{0}$ by $t_{0}^{\prime}$. We set $t_{0}^{\prime}=d$. For $\varepsilon<1 / 4$, we take $\delta>0$ such that $t_{0}^{\prime}+\delta \leqslant b_{0}$ and $\left|\widehat{r}\left(t, t_{0}^{\prime}\right)-\widehat{r}\left(t_{0}^{\prime}, t_{0}^{\prime}\right)\right|<\varepsilon$ for all $t \in \mathcal{I}_{\delta, t_{0}^{\prime}}$. We fix the point $t_{1}=t_{0}^{\prime}-\delta / 8$. Then the inequality

$$
\left|\widehat{r}\left(t, t_{0}^{\prime}\right)-\widehat{r}\left(t_{1}, t_{0}^{\prime}\right)\right| \leqslant\left|\widehat{r}\left(t, t_{0}^{\prime}\right)-\widehat{r}\left(t_{0}^{\prime}, t_{0}^{\prime}\right)\right|+\left|\widehat{r}\left(t_{0}^{\prime}, t_{0}^{\prime}\right)-\widehat{r}\left(t_{1}, t_{0}^{\prime}\right)\right|<2 \varepsilon<1 / 2
$$

holds for all $t$ such that $\left|t-t_{1}\right| \leqslant \delta / 2$.

We introduce the operator $B$ in the space $\widetilde{C}\left(\mathcal{I}_{\delta / 2, t_{1}}\right)$ by the equality

$$
(B y)(t)=\int_{t_{1}}^{t} d \mathbf{r}\left(\xi, t_{0}^{\prime}\right) y(\xi)=\int_{t_{1}}^{t} \Psi_{\mathbf{p}}(\xi) y(\xi) d \widehat{r}\left(\xi, t_{0}^{\prime}\right) .
$$

It follows from 12 that $\sup _{t \in \mathcal{I}_{\delta / 2, t_{1}}}\|(B y)(t)\| \leqslant(1 / 2) \sup _{t \in \mathcal{I}_{\delta / 2, t_{1}}}\|y(t)\|$. Hence the operator $E-B$ has the bounded everywhere defined inverse operator in $\widetilde{C}\left(\mathcal{I}_{\delta / 2, t_{1}}\right)$. We set $v=(E-B)^{-1} z_{1}, w=(E-B)^{-1} z_{2}$, where $z_{1}(t)=$ $=u\left(t_{1}\right)-g\left(t_{1}\right)+g(t), z_{2}(t)=z_{1}(t)+\varphi(t), \varphi(t)=\widetilde{\mathbf{r}}_{t_{0}^{\prime}}(t) v\left(t_{0}^{\prime}\right)$, and as above, $\widetilde{\mathbf{r}}_{t_{0}^{\prime}}(t)=0$ for $t \leqslant t_{0}^{\prime}$ and $\widetilde{\mathbf{r}}_{t_{0}^{\prime}}(t)=\mathbf{p}\left(\left\{t_{0}^{\prime}\right\}\right)$ for $t>t_{0}^{\prime}$. Then the equalities

$$
\begin{array}{r}
v(t)=\int_{t_{1}}^{t} \Psi_{\mathbf{p}}(\xi) v(\xi) d \widehat{r}\left(\xi, t_{0}^{\prime}\right)+u\left(t_{1}\right)-g\left(t_{1}\right)+g(t), \\
w(t)=\int_{t_{1}}^{t} \Psi_{\mathbf{p}}(\xi) w(\xi) d \widehat{r}\left(\xi, t_{0}^{\prime}\right)+u\left(t_{1}\right)-g\left(t_{1}\right)+\varphi(t)+g(t)
\end{array}
$$

hold for all $t \in\left[t_{1}-\delta / 2, t_{1}+\delta / 2\right]$. Using (14), we have

$$
w(t)=\int_{t_{1}}^{t} \Psi_{\mathbf{p}}(\xi) w(\xi) d \widehat{r}\left(\xi, t_{0}^{\prime}\right)+u\left(t_{1}\right)-g\left(t_{1}\right)+g(t)
$$


for all $t \in\left[t_{1}-\delta / 2, t_{0}^{\prime}\right]$. It follows from $(12)$ that the equation

$$
y(t)=\int_{t_{1}}^{t} \Psi_{\mathbf{p}}(\xi) y(\xi) d \widehat{r}\left(\xi, t_{0}^{\prime}\right)+\widetilde{g}(t), \quad \widetilde{g} \in \widetilde{C}\left[a, b_{0}\right],
$$

has a unique solution on the interval $\left(t_{1}-\delta / 8, t_{0}^{\prime}\right)$. Using (13), 15, we obtain that $v(t)=w(t)$ for all $t \in\left(t_{1}-\delta / 8, t_{0}^{\prime}\right)$. Therefore, $w\left(t_{0}^{\prime}\right)=v\left(t_{0}^{\prime}\right)$. Moreover, using 14 and the equality $\widehat{\rho}(t)=\widehat{r}\left(t, t_{0}^{\prime}\right)+\widehat{r}_{t_{0}^{\prime}}(t)$, we get

$$
w(t)=\int_{t_{1}}^{t}(d \mathbf{p}) w(s)+u\left(t_{1}\right)-g\left(t_{1}\right)+g(t)
$$

for all $t \in \mathcal{I}_{\delta / 2, t_{1}}$ (i.e., for all $t$ such that $t_{1}-\delta / 2 \leqslant t \leqslant t_{0}^{\prime}+(3 / 8) \delta$ ).

The function $u$ is the solution of equation (1) on $\left[t_{0}-\delta, t_{0}^{\prime}\right)$. Therefore,

$$
u(t)=\int_{t_{0}}^{t}(d \mathbf{p}) u(\xi)+g(t)=u\left(t_{1}\right)-g\left(t_{1}\right)+\int_{t_{1}}^{t}(d \mathbf{p}) u(\xi)+g(t),
$$

where $t_{0}-\delta \leqslant t<t_{0}^{\prime}$. Taking into account the equality $\widehat{r}\left(t, t_{0}^{\prime}\right)=\widehat{\rho}(t)$ for $t \leqslant t_{0}^{\prime}$ and using (18), we obtain

$$
u(t)=\int_{t_{1}}^{t} \Psi_{\mathbf{p}}(\xi) u(\xi) d \widehat{r}\left(\xi, t_{0}^{\prime}\right)+u\left(t_{1}\right)-g\left(t_{1}\right)+g(t) .
$$

It follows from (13), 15), 19), and the uniqueness of the solution of (16) that the functions $u, v, w$ coincide on the interval $\left(t_{1}-\delta / 8, t_{0}^{\prime}\right)$. This implies that there exists $\lim _{t \rightarrow t_{0}^{\prime}-0} u(t)=w\left(t_{0}^{\prime}\right)=v\left(t_{0}^{\prime}\right)$. By $\widetilde{u}$ denote a function such that $\widetilde{u}$ is equal to $u$ on $\left[t_{0}-\delta\left(t_{0}\right), t_{0}^{\prime}\right)$ and $\widetilde{u}$ is equal to $w$ on $\left[t_{0}^{\prime}, t_{0}^{\prime}+(3 / 8) \delta\right]$. We claim that $\widetilde{u}$ is the solution of (1). Indeed, since $u$ is the solution of (1) for $t<t_{0}^{\prime}$, we see that $\widetilde{u}$ is the solution of (1) for $t<t_{0}^{\prime}$. Suppose $t_{1} \leqslant t \leqslant t_{0}^{\prime}+(3 / 8) \delta$. Using (17), 18), we get

$$
\begin{aligned}
\int_{t_{0}}^{t}(d \mathbf{p}) \widetilde{u}(\xi)+g(t)= & \int_{t_{0}}^{t_{1}}(d \mathbf{p}) u(\xi)+\int_{t_{1}}^{t}(d \mathbf{p}) w(\xi)+g(t)= \\
& =\left(u\left(t_{1}\right)-g\left(t_{1}\right)\right)+w(t)-u\left(t_{1}\right)+g\left(t_{1}\right)=\widetilde{u}(t) .
\end{aligned}
$$

So $\widetilde{u}$ is a solution of (1) and $\widetilde{u}$ is an extension of $u$ to $\left[t_{0}^{\prime}, t_{0}^{\prime}+(3 / 8) \delta\right]$. 
Let us show the uniqueness of the solution of equation (1). Suppose that $u_{1}, u_{2}$ are two solutions of (1). As shown above, $u_{1}(t)=u_{2}(t)$ for all $t \in \mathcal{I}_{\delta, t_{0}}$. By $T$ denote the supremum of the set $t$ such that $u_{1}(t)=u_{2}(t)$ for all $t<T$. Since $u_{1}, u_{2}$ are continuous from the left, we have $u_{1}(T)=$ $=u_{2}(T)$. For $t>T$, we obtain

$$
u_{i}(t)=x_{1}-g(T)+\int_{T}^{t}(d \mathbf{p}) u_{i}(\xi)+g(t), x_{1}=u_{1}(T)=u_{2}(T), i=1,2 .
$$

As we have shown above, there exists a unique solution of equation 20 on an interval $\mathcal{I}_{\delta, T}$. Consequently, $T=b_{0}$. The Theorem is proved.

Corollary 1. If $t_{0}=a$, then for any function $g \in \widetilde{C}\left[a, b_{0}\right]$ there exists a unique solution of equation (1) belonging to the space $\widetilde{C}\left[a, b_{0}\right]$.

Note that Theorem 1 corrects the error made by the author in the article [7] in the proof of the similar theorem.

Remark 1. In the proof of Theorem 1, we take $\delta$ such that $\varepsilon<1 / 2$ in inequality (9). Then $\left\|(E-A)^{-1}\right\|_{\widetilde{C}\left(\mathcal{I}_{\delta, t_{0}}\right)} \leqslant 2$. Using (8), (11), we get

$$
\|y\|_{\widetilde{C}\left(\mathcal{I}_{\delta, t_{0}}\right)} \leqslant 2\left(1+\mathbf{V}_{\left[a, b_{0}\right]}(\mathbf{p})\right)\|g\|_{\widetilde{C}\left(\left[a, b_{0}\right]\right)} .
$$

Remark 2. For $t<t_{0}$, the solution of equation (1) can be non-unique. In addition, generally, the solution can be non-extendable to the left.

We give examples to corroborate Remark 2, Suppose $H=\mathbb{C}$. Let $\mathbf{p}$ be the measure generated by a function $\widehat{p}$ on the segment $[0,2]$ (i.e., $\mathbf{p}([\alpha, \beta))=\widehat{p}(\beta)-\widehat{p}(\alpha), 0 \leqslant \alpha \leqslant \beta \leqslant 2)$, where $\widehat{p}(t)=0$ for $0 \leqslant t \leqslant 1$ and $\widehat{p}(t)=-1$ for $1<t \leqslant 2$. We consider the equation $y=\int_{2}^{t} y d \mathbf{p}$. This equation has solutions $y=y_{1}, y=y_{2}$, where $y_{1}(t)=0$ for all $t, y_{2}(t)=1$ for $0 \leqslant t \leqslant 1$ and $y_{2}(t)=0$ for $1<t \leqslant 2$. Further, consider the equation $y=1+\int_{2}^{t} y d \mathbf{p}$. The function $y=1$ is a solution of this equation for $1<t \leqslant 2$. We claim that this solution can not be extended to the left. Indeed, assume the converse, i.e., suppose that the solution is extended to the left. Then $y(1)=1-\int y(s) d \mathbf{p}$. Hence $y(1)=1+y(1)$. This equality is impossible. 
Lemma 1. For any point $t_{0} \in\left(a, b_{0}\right]$ there exists $\delta=\delta\left(t_{0}\right)$ such that for all $s \in\left[t_{0}-\delta, t_{0}\right]$ the equation

$$
y_{s}(t)=\int_{s}^{t} d \mathbf{p}(\xi) y_{s}(\xi)+h(t), \quad h \in \widetilde{C}\left[a, b_{0}\right],
$$

has a unique solution in $\widetilde{C}\left[t_{0}-\delta, b_{0}\right]$. This solution satisfies the inequality

$$
\left\|y_{s}\right\|_{\widetilde{C}\left[t_{0}-\delta, t_{0}\right]} \leqslant 8\left(1+\mathbf{V}_{\left[a, b_{0}\right]}(\mathbf{p})\right)\|h\|_{\widetilde{C}\left(\left[a, b_{0}\right]\right)}
$$

for all $s \in\left[t_{0}-\delta, t_{0}\right]$.

Proof. According to Theorem 1, there exists $\delta_{s}>0$ such that equation (22) has a unique solution in the space $\widetilde{C}\left[s-\delta_{s}, b_{0}\right]$. In the proof of Theorem 1, for the point $t_{0}$ we take $\delta$ such that $\varepsilon<1 / 4$ in (9), 10. Then

$$
\begin{gathered}
\int_{s}^{t} d \mathbf{p}(\xi) y_{s}(\xi)+h(t)=\int_{s}^{t_{0}} d \mathbf{p}(\xi) y_{s}(\xi)+h\left(t_{0}\right)+\int_{t_{0}}^{t} d \mathbf{p}(\xi) y_{s}(\xi)+h(t)-h\left(t_{0}\right)= \\
=y_{s}\left(t_{0}\right)+\int_{t_{0}}^{t} d \mathbf{p}(\xi) y_{s}(\xi)+h(t)-h\left(t_{0}\right) . \quad
\end{gathered}
$$

This implies that every solution of equation 22 is a solution of equation (1) in which $g(t)=y_{s}\left(t_{0}\right)+h(t)-h\left(t_{0}\right)$. Equations (1), (22) have unique solutions. Hence equation $(22)$ has a unique solution in $C\left[t_{0}-\delta, b_{0}\right]$.

We shall prove that inequality $(23)$ is satisfied. Inequality 23 follows from (21) for $s=t_{0}$. Suppose $s \in\left[t_{0}-\delta, t_{0}\right)$. We use the notation from the proof of Theorem 1. It follows from (10) and the inequality $\varepsilon<1 / 4$ that

$$
\left|\widehat{r}\left(t, t_{0}\right)-\widehat{r}\left(s, t_{0}\right)\right| \leqslant\left|\widehat{r}\left(t, t_{0}\right)-\widehat{r}\left(t_{0}, t_{0}\right)\right|+\left|\widehat{r}\left(t_{0}, t_{0}\right)-\widehat{r}\left(s, t_{0}\right)\right|<2 \varepsilon<1 / 2,
$$

where $s, t \in\left[t_{0}-\delta, t_{0}\right]$.

We define operators $B_{s}\left(t_{0}-\delta \leqslant s<t_{0}\right)$ in the space $\widetilde{C}\left[t_{0}-\delta, t_{0}\right]$ by the equality

$$
\left(B_{s} u\right)(t)=\int_{s}^{t} \Psi_{\mathbf{p}}(\xi) u(\xi) d \widehat{r}\left(\xi, t_{0}\right) .
$$

It follows from $(25),(26)$ that $\left\|B_{s}\right\| \leqslant 1 / 2$. Consequently, for all $s \in$ $\in\left[t_{0}-\delta, t_{0}\right)$ there exists a bounded everywhere defined operator $\left(E-B_{s}\right)^{-1}$ 
with the norm $\left\|\left(E-B_{s}\right)^{-1}\right\| \leqslant 2$. We denote $y_{s}=\left(E-B_{s}\right)^{-1} h_{1}$, where $h_{1}$ is the restriction of function $h$ to $\left[t_{0}-\delta, t_{0}\right]$. Then

$$
y_{s}(t)=\int_{s}^{t} \Psi_{\mathbf{p}}(\xi) y_{s}(\xi) d \widehat{r}\left(\xi, t_{0}\right)+h(t) .
$$

In the proof of Theorem 1, we have established that the equality $\widehat{r}\left(t, t_{0}\right)=\widehat{\rho}(t)-\widehat{r}_{t_{0}}(t)$ holds, where $\widehat{r}_{t_{0}}(t)=0$ for $t \leqslant t_{0}$ and $\widehat{r}_{t_{0}}(t)=\widehat{\rho}_{t_{0}}$ for $t>t_{0}\left(\widehat{\rho}_{t_{0}}\right.$ is the jump of the function $\widehat{\rho}$ at the point $\left.t_{0}\right)$. Using (27), we get

$$
y_{s}(t)=\int_{s}^{t} \Psi_{\mathbf{p}}(\xi) y(\xi) d \widehat{\rho}(\xi)+h(t)=\int_{s}^{t} d \mathbf{p}(\xi) y_{s}(\xi)+h(t) .
$$

It follows from Theorem 1 that the function $y_{s}$ is the solution of equation (22) on $\left[t_{0}-\delta, b_{0}\right]$. Using (27), we obtain

$$
\left\|y_{s}\right\|_{\widetilde{C}\left(\left[t_{0}-\delta, t_{0}\right]\right)} \leqslant\left\|\left(E-B_{s}\right)^{-1} h_{1}\right\| \leqslant 2\|h\|_{\widetilde{C}\left(\left[a, b_{0}\right]\right)}
$$

for all $s \in\left[t_{0}-\delta, t_{0}\right)$. Hence $\left\|y_{s}\left(t_{0}\right)\right\| \leqslant 2\|h\|_{\widetilde{C}\left(\left[a, b_{0}\right]\right)}$. It follows from (21), (24), (28) that inequality 23 holds. The Lemma is proved.

3. Family of evolution operators. We consider the equation (2). Theorem 1 implies that equation (2) has a unique solution $y_{s} \in \widetilde{C}\left[s-\delta, b_{0}\right]$ for all $c \in H$, where $\delta=\delta(s)>0$ is sufficiently small $(\delta(s)=0$ for $s=a)$. It follows from the proof of Theorem 1 that $\delta(s)$ independent of $g(t)=c$. Let $U(t, s)$ be the operator taking each element $c \in H$ to the value of solution $y_{s}(t)$ of (2). The function $t \rightarrow U(t, s) c$ is the solution of the equation

$$
U(t, s) c=c+\int_{s}^{t} d \mathbf{p}(\tau) U(\tau, s) c .
$$

Remark 3. Since $\mathbf{p}(\Delta)=0$ for all Borel sets $\Delta \subset\left(b, b_{0}\right]$, we see that solution $y_{s}$ of equation (2) is a constant function on $\left(b, b_{0}\right]$ and $y_{s}(t)=$ $=\lim _{\xi \rightarrow b+0} y_{s}(\xi)$ for $t>b$.

Lemma 2. The operator $U(t, s)$ is bounded for fixed $t, s$.

Proof. Equation (2) is a special case of equation (1) for $g(t)=c$ and $t_{0}=s$. Using 21$)$, we get $\|U(t, s) c\|_{\widetilde{C}\left(\mathcal{I}_{\delta, s}\right)} \leqslant k\|c\|$. This implies that 
the operator $U(t, s)$ is bounded for $t \in \mathcal{I}_{\delta, s}$. By $T=T(s)$ denote the supremum of the set $t \geqslant s$ such that the operator $U(\tau, s)$ is bounded for all $s \leqslant \tau \leqslant t$. Since a solution of equation (2) is continuous from the left, we see that the operator $U(T, s)$ is bounded. Using 299 , we obtain

$$
U(t, s) c=\int_{T}^{t} d \mathbf{p}(\tau) U(\tau, s) c+\int_{s}^{T} d \mathbf{p}(\tau) U(\tau, s) c+c, \quad t>T .
$$

Hence the function $y(t)=U(t, s) c$ is a solution of equation (1) for $t_{0}=T, g(t)=\int_{s}^{T} d \mathbf{p}(\tau) U(\tau, s) c+c$. It follows from 21 that the operator $U(t, s)$ is bounded for $t \in \mathcal{I}_{\delta, T}$. Therefore $T=b_{0}$. The Lemma is proved.

Lemma 3. The function $t \rightarrow U(t, s)$ is continuous from the left with respect to the uniform operator topology.

Proof. Using (29), we get

$$
U\left(t_{1}, s\right) c-U(t, s) c=\int_{\left[t, t_{1}\right)} d \mathbf{p}(\xi) U(\xi, s) c=\int_{\left[t, t_{1}\right)} \Psi_{\mathbf{p}}(\xi) U(\xi, s) c d \rho(\xi)
$$

for $t<t_{1}$ and $t, t_{1} \in\left[s-\delta, b_{0}\right], \delta=\delta(s)>0$ (if $s=a$, then $t, t_{1} \in\left[a, b_{0}\right]$ ). It follows from the Banach-Steinhaus Theorem and Theorem 1 that the function $t \rightarrow\|U(t, s)\|$ is bounded. Using (30), we obtain

$$
\left\|U\left(t_{1}, s\right) c-U(t, s) c\right\| \leqslant \int_{\left[t, t_{1}\right)}\left\|\Psi_{\mathbf{p}}(\xi)\right\|\|U(\xi, s)\|\|c\| d \rho(\xi) \leqslant k\|c\| \int_{\left[t, t_{1}\right)} d \rho(\xi) .
$$

Hence $\left\|U\left(t_{1}, s\right)-U(t, s)\right\| \rightarrow 0$ as $t \rightarrow t_{1}-0$. The Lemma is proved.

Lemma 4. The equality

$$
U(t, \tau) U(\tau, s)=U(t, s) .
$$

holds for all points $s, \tau, t \in\left[a, b_{0}\right]$ such that $s \leqslant \tau \leqslant t$. Moreover, for any point $t_{0} \in\left(a, b_{0}\right]$ there exists $\delta=\delta\left(t_{0}\right)>0$ such that equality (31) is valid in the following cases: 1) $\tau, s \in\left[t_{0}-\delta, t_{0}\right], t \geqslant \tau$; 2) $\tau, s, t \in\left[t_{0}-\delta, t_{0}\right]$.

Proof. We denote $y(t)=U(t, s) c, z(t)=U(t, \tau) y(\tau)(c \in H)$. It follows from (29) and Lemma 1 that the functions $y, z$ exist in all cases mentioned 
in Lemma 4. Therefore,

$$
y(t)=c+\int_{s}^{t}(d \mathbf{p}) y(\xi)=c+\int_{s}^{\tau}(d \mathbf{p}) y(\xi)+\int_{\tau}^{t}(d \mathbf{p}) y(\xi)=y(\tau)+\int_{\tau}^{t}(d \mathbf{p}) y(\xi) .
$$

On the other hand, we have $z(t)=y(\tau)+\int_{\tau}^{t}(d \mathbf{p}) z(\xi)$. Using Theorem 1 , we get $y(t)=z(t)$. Now the desired statement follows from the equality $y(\tau)=U(\tau, s) c$. The Lemma is proved.

Lemma 5. The function $(t, s) \rightarrow\|U(t, s)\|$ is bounded in the triangle $\left\{(t, s): t, s \in\left[a, b_{0}\right], t \geqslant s\right\}$.

Proof. Equation (2) is a special case of equation (22). It follows from Lemmas 1, 4 that for any point $t_{0} \in\left[a, b_{0}\right]$ there exists $\delta_{0}=\delta\left(t_{0}\right)>0$ such that the inequality

$$
\|U(t, s)\| \leqslant\left\|U\left(t, t_{0}\right)\right\|\left\|U\left(t_{0}, s\right)\right\| \leqslant 64\left(1+\mathbf{V}_{\left[a, b_{0}\right]}(\mathbf{p})\right)^{2}
$$

holds for all $t, s \in \mathcal{I}_{\delta_{0}, t_{0}}, t \geqslant s$. We consider a covering of the segment $\left[a, b_{0}\right]$ by open intervals such that inequality (32) holds in each interval. From this covering, we choose a finite covering by intervals denoted by $\mathcal{I}_{k}, k=1, \ldots, n$. We consider all possible finite non-empty intersections of these intervals $\mathcal{I}_{k}$. We add the intervals $\mathcal{I}_{k}$ to these intersections. So we obtain intervals $\mathcal{J}_{i}, i=1, \ldots, r$. Let $s_{i}$ be the centre of $\mathcal{J}_{i}$. We enumerate the number $s_{i}$ in ascending order, i.e., $s_{i} \leqslant s_{i+1}$. We claim that for any two neighboring points $s_{i}, s_{i+1}\left(s_{i} \neq s_{i+1}\right)$ there exists an interval $\mathcal{J}_{m}$ such that $\mathcal{J}_{m} \supset\left[s_{i}, s_{i+1}\right]$. Indeed, let $\mathcal{J}_{m}$ be the interval containing the point $s_{i}$ and having the greatest right boundary $\beta_{m}$. In the case $\beta_{m} \leqslant s_{i+1}$, we choose an interval $\mathcal{J}_{q}$ such that $s_{i+1} \in \mathcal{J}_{q}, s_{i} \notin \mathcal{J}_{q}$, and $\mathcal{J}_{q}$ has the smallest left boundary. Then $\mathcal{I}_{l} \cap \mathcal{I}_{q} \neq \varnothing$, and the middle of interval $\mathcal{I}_{l} \cap \mathcal{I}_{q}$ lies between points $s_{i}, s_{i+1}$. This contradicts the assumption that $s_{i}, s_{i+1}$ are neighboring points.

Suppose $s, t \in\left[a, b_{0}\right], s \leqslant t$. By $s_{m}, s_{m+1}, s_{j}, s_{j+1}$ we denote the points defined above such that $s_{m} \leqslant s<s_{m+1}, s_{j} \leqslant t<s_{j+1}$. Using Lemma 4, we get $U(t, s)=U\left(t, s_{j}\right) \cdots U\left(s_{m+2}, s_{m+1}\right) U\left(s_{m+1}, s\right)$. Inequality (32) holds on all segments $\left[s_{i}, s_{i+1}\right]$. Consequently,

$$
\|U(t, s)\| \leqslant\left\|U\left(t, s_{j}\right)\right\| \cdots\left\|U\left(s_{m+1}, s\right)\right\| \leqslant 64^{r}\left(1+\mathbf{V}_{\left[a, b_{0}\right]}(\mathbf{p})\right)^{2 r} .
$$


The Lemma is proved.

Consider the equation

$$
y(t)=\int_{a}^{t}(d \mathbf{p}) y(s)+g(t), \quad a \leqslant t \leqslant b_{0}, \quad g \in \widetilde{C}\left[a, b_{0}\right] .
$$

We define an operator $\mathcal{P}$ in the space $\widetilde{C}\left[a, b_{0}\right]$ by the equality

$$
(\mathcal{P} u)(t)=\int_{a}^{t}(d \mathbf{p}) u(s), \quad u \in \widetilde{C}\left[a, b_{0}\right], \quad a \leqslant t \leqslant b_{0} .
$$

Using (6), we get $\|\mathcal{P} u\| \leqslant \mathbf{V}_{[a, b]}(\mathbf{p}) \sup _{t \in\left[a, b_{0}\right]}\|u(t)\|$. Hence the operator $\mathcal{P}$ is bounded. By Corollary 1 , it follows that there exists an everywhere defined operator $(E-\mathcal{P})^{-1}$. The operator $(E-\mathcal{P})^{-1}$ is bounded. The solution of equation (33) has the form $y=(E-\mathcal{P})^{-1} g$.

Below we assume that the function $g$ has the form

$$
g(t)=\int_{a}^{t}(d \mathbf{q}) f(s)
$$

where $\mathbf{q}$ is an operator measure with the bounded variation on $[a, b]$; the function $f$ is integrable with respect to $\mathbf{q}$. We extend the measure $\mathbf{q}$ to the segment $\left[a, b_{0}\right]$, letting $\mathbf{q}(\Delta)=0$ for each Borel sets $\Delta \subset\left[a, b_{0}\right] \backslash[a, b]$.

Lemma 6. Suppose that the function $g$ has form (34), and $y=$ $=(E-\mathcal{P})^{-1} g$, and $\mathbf{p}, \mathbf{q}$ are measures with bounded variations, and $\mathbf{p}, \mathbf{q}$ have no common atoms consisting of one point, i.e., on the segment $\left[a, b_{0}\right]$ there are no singleton sets $\{\tau\}$ such that the inequalities $\mathbf{p}(\{\tau\}) \neq 0$, $\mathbf{q}(\{\tau\}) \neq 0$ hold together. Then

$$
y(t)=\int_{a}^{t} U(t, s) d \mathbf{q}(s) f(s) .
$$

Proof. We substitute function (35) for $y$ in the following integral. Then

$$
\int_{a}^{t} d \mathbf{p}(\xi) y(\xi)=\int_{[a, t)} d \mathbf{p}(\xi) \int_{[a, \xi)} U(\xi, \tau) d \mathbf{q}(\tau) f(\tau) .
$$


We change the limits of integration. Then

$$
\begin{gathered}
\int_{[a, t)} d \mathbf{p}(\xi) \int_{[a, \xi)} U(\xi, \tau) d \mathbf{q}(\tau) f(\tau)= \\
\left.=\iint_{[a, t)} d \mathbf{p}(\xi) U(\xi, \tau)\right) d \mathbf{q}(\tau) f(\tau)= \\
\left.=\int_{[a, t)}\left(\int_{[\tau, t)} d \mathbf{p}(\xi) U(\xi, \tau)\right) d \mathbf{q}(\tau) f(\tau)-\iint_{[a, t)} d \mathbf{p}(\xi) U(\xi, \tau)\right) d \mathbf{q}(\tau) f(\tau) .
\end{gathered}
$$

We consider the function $h(\tau)=\int_{\{\tau\}} d \mathbf{p}(\xi) U(\xi, \tau)$. If $\mathbf{p}(\{\tau\})=0$, then $h(\tau)=0$. By $T_{1}$ denote the set of points $\tau$ such that $\mathbf{p}(\{\tau\}) \neq 0$. The set $T_{1}$ is countable or finite. It follows from the conditions of the Lemma that $\mathbf{q}(\{\tau\})=0$ if $\tau \in T_{1}$. Therefore, $\int_{[a, t)} h(\tau) d \mathbf{q}(\tau) f(\tau)=0$. Consequently,

$$
\begin{aligned}
\int_{a}^{t} d \mathbf{p}(\xi) y(\xi)=\int_{[a, t)}\left(\int_{[\tau, t)} d \mathbf{p}(\xi) U(\xi, \tau)\right) & d \mathbf{q}(\tau) f(\tau)= \\
& =\int_{[a, t)}(U(t, \tau)-E) d \mathbf{q}(\tau) f(\tau) .
\end{aligned}
$$

We substitute function (35) for $y$ in the right-hand side of equation (33). Using (36), we get

$$
\begin{aligned}
& \int_{a}^{t} d \mathbf{p}(\xi) y(\xi)+\int_{a}^{t} d \mathbf{q}(\tau) f(\tau)= \\
& \quad=\int_{a}^{t}(U(t, \tau)-E) d \mathbf{q}(\tau) f(\tau)+\int_{a}^{t} d \mathbf{q}(\tau) f(\tau)=y(t) .
\end{aligned}
$$

So function 35 is the solution of $(33)$. Now the desired statement follows from the uniqueness of the solution of (33). The Lemma is proved.

Using Lemma 6 and Corollary 1, we obtain Theorem 2 (see the Introduction). 
4. Maximal relation. Let $\mathbf{B}_{1}, \mathbf{B}_{2}$ be Banach spaces. A linear relation $\mathrm{T}$ is understood as any linear manifold $\mathrm{T} \subset \mathbf{B}_{1} \times \mathbf{B}_{2}$. The terminology on the linear relations can be found, for example, in [1, 2, , 8]. In what follows we make use of the following notations: $\{\cdot, \cdot\}$ is an ordered pair; ker $\mathrm{T}$ is a set of elements $x \in \mathbf{B}_{1}$ such that $\{x, 0\} \in \mathrm{T}$; Ker $\mathrm{T}$ is a set of ordered pairs of the form $\{x, 0\} \in \mathrm{T} ; \mathcal{D}(\mathrm{T})$ is the domain of $\mathrm{T} ; \mathcal{R}(\mathrm{T})$ is the range of $\mathrm{T} ; \mathrm{T}^{-1}$ is the inverse relation for $\mathrm{T}$, i.e., the relation formed by the pairs $\left\{x^{\prime}, x\right\}$, where $\left\{x, x^{\prime}\right\} \in \mathrm{T}$. A relation $\mathrm{T}$ is called surjective if $\mathcal{R}(\mathrm{T})=\mathbf{B}_{2}$. A relation $\mathrm{T}$ is called invertible or injective if ker $\mathrm{T}=\{0\}$ (i.e., the relation $\mathrm{T}^{-1}$ is an operator); it is called continuously invertible if it is closed, invertible, and surjective (i.e., $\mathrm{T}^{-1}$ is a bounded everywhere defined operator). Linear operators are treated as linear relations, this is why the notation $\left\{x_{1}, x_{2}\right\} \in \mathrm{T}$ is used also for an operator $\mathrm{T}$. Since all considered relations are linear, we shall often omit the word "linear".

Let $\mathbf{m}$ be a non-negative operator-valued measure defined on Borel sets $\Delta \subset[a, b]$ and taking values in the set of linear bounded non-negative operators acting in the space $H$. The measure $\mathbf{m}$ is assumed to have a bounded variation on $[a, b]$. We extend the measure $\mathbf{m}$ to the segment $\left[a, b_{0}\right]$, letting $\mathbf{m}(\Delta)=0$ for each Borel sets $\Delta \subset\left[a, b_{0}\right] \backslash[a, b]$.

We introduce the quasi-scalar product $(x, y)_{\mathbf{m}}=\int_{a}^{b_{0}}((d \mathbf{m}) x(t), y(t))$ on a set of step-like functions with values in $H$ defined on the segment $\left[a, b_{0}\right]$. Identifying with zero functions $y$ obeying $(y, y)_{\mathbf{m}}=0$ and making the completion, we arrive at the Hilbert space denoted by $L_{2}(H, d \mathbf{m} ; a, b)=\mathfrak{H}$. The elements of $\mathfrak{H}$ are the classes of functions identified with respect to the norm $\|y\|_{\mathbf{m}}=(y, y)_{\mathbf{m}}^{1 / 2}$. In order not to complicate the terminology, the class of functions with a representative $y$ is indicated by the same symbol and we write $y \in \mathfrak{H}$. The equalities of the functions in $\mathfrak{H}$ are understood as the equality for associated equivalence classes.

We consider equation (3). Let $L^{\prime}$ be a relation consisting of the pairs $\{\tilde{y}, \tilde{f}\} \in \mathfrak{H} \times \mathfrak{H}$ satisfying the condition: for each pair $\{\tilde{y}, \tilde{f}\}$ there exists a pair $\{y, f\}$ such that the pairs $\{\tilde{y}, \tilde{f}\},\{y, f\}$ are identical in $\mathfrak{H} \times \mathfrak{H}$, and equality (3) holds on $\left[a, b_{0}\right]$. By $L$ we denote the closure of $L^{\prime}$ and we call $L$ the maximal relation generated by equation (3). Generally speaking, relation $L$ is not an operator since function $y$ can happen to be identified with zero in $\mathfrak{H}$, while $f$ is non-zero.

Remark 4. Since the equalities $\mathbf{p}(\Delta)=\mathbf{m}(\Delta)=0$ hold for each Borel set 
$\Delta \subset\left[a, b_{0}\right] \backslash[a, b]$, we have $y\left(b_{0}\right)=\lim _{t \rightarrow b+0} y(t)$, where $y$ is a solution of $(3)$.

Let $Q_{0}$ be a set of elements $x \in H$ such that the function $t \rightarrow U(t, a) x$ is identified with zero in $\mathfrak{H}$. We put $Q=H \ominus Q_{0}$. On a linear manifold $Q$, we introduce the norm

$$
\|c\|_{-}=\|U(\cdot, a)\|_{\mathbf{m}}=\left(\int_{a}^{b_{0}}(d \mathbf{m}(\xi) U(\xi, a) c, U(\xi, a) c)\right)^{1 / 2}, c \in Q .
$$

Using formula (4), we get

$$
\|c\|_{-}=\left(\int_{a}^{b_{0}}\left(\Psi_{\mathbf{m}}(s) U(s, a) c, U(s, a) c\right) d \rho\right)^{1 / 2} \leqslant \gamma\|c\|, \quad c \in Q .
$$

By $Q_{-}$denote the completion of $Q$ with respect to norm (37). It follows from $(38)$ that the space $Q_{-}$can be treated as a space with a negative norm with respect to $Q$ [4, Ch. 1]. Suppose that a sequence $\left\{c_{n}\right\}\left(c_{n} \in Q\right)$ converges in $Q_{-}$to $c_{0} \in Q_{-}$. Then a sequence $\left\{U(\cdot, a) c_{n}\right\}$ is fundamental in $\mathfrak{H}$ and hence, it converges to some element in $\mathfrak{H}$. By $U(\cdot, a) c_{0}$ we denote this element and $\mathcal{U}$ stands for the operator $c \rightarrow U(\cdot, a) c$, where $c \in Q_{-}$.

Theorem 3. Suppose that $\mathbf{m}, \mathbf{p}$ are measures with bounded variations and $\mathbf{m}, \mathbf{p}$ have no common atoms consisting of one point. A pair $\{\tilde{y}, \tilde{f}\} \in$ $\in \mathfrak{H} \times \mathfrak{H}$ belongs to the relation $L$ if and only if there exists a pair $\{y, f\}$ such that the pairs $\{\tilde{y}, \tilde{f}\},\{y, f\}$ are identified in $\mathfrak{H} \times \mathfrak{H}$ with each other and the equality

$$
y(t)=U(t, a) c+\int_{a}^{t} U(t, s) d \mathbf{m}(s) f(s)
$$

holds, where $c \in Q_{-}$.

Proof. Suppose that equality $(39)$ holds. It follows from the definition of the relation $L$ and Theorem 2 that $\{y, f\} \in L^{\prime} \subset L$ for $c \in Q$. Since the relation $L$ is closed, we see that $\{y, f\} \in L$ for $c \in Q_{-}$.

Conversely, suppose that $\{y, f\} \in L$. Then there exists a sequence of pairs $\left\{y_{n}, f_{n}\right\} \in L^{\prime}$ such that it converges to $\{y, f\}$ in $\mathfrak{H} \times \mathfrak{H}$. Using Theorem 2, we obtain 


$$
y_{n}(t)=U(t, a) c_{n}+\int_{a}^{t} U(t, s) d \mathbf{m}(s) f_{n}(s),
$$

where $c_{n} \in Q$. We put

$$
F_{n}(t)=\int_{a}^{t} U(t, s) d \mathbf{m}(s) f_{n}(s), \quad F(t)=\int_{a}^{t} U(t, s) d \mathbf{m}(s) f(s) .
$$

Since $f_{n} \rightarrow f$ in $\mathfrak{H}$ as $n \rightarrow \infty$, we have $F_{n} \rightarrow F$ in $\mathfrak{H}$. The sequence $\left\{y_{n}\right\}$ converges to $y$ in $\mathfrak{H}$. Hence there exists an element $c \in Q_{-}$such that the sequence $\left\{U(t, a) c_{n}\right\}$ converges to $\{U(t, a) c\}$ in $\mathfrak{H}$. Thus equality (39) holds. The Theorem is proved.

Corollary 2. The operator $\mathcal{U}$ is a continuous one-to-one mapping of $Q_{-}$onto ker $L$.

5. Spaces of boundary values and states of linear relations. In what follows we shall make use of a space of boundary values (SBV) for the maximal relation $L$. Let $\mathbf{B}_{1}, \mathbf{B}_{2}, B_{1}, B_{2}$ be Banach spaces, $T \subset \mathbf{B}_{1} \times \mathbf{B}_{2}$ be a closed linear relation, $\delta: T \rightarrow B_{1} \times B_{2}$ be a linear operator, $\delta_{j}=P_{j} \delta$, $j=1,2\left(P_{j}\right.$ indicates the natural projection onto a set $G_{j}$ in the Cartesian product $\left.G=G_{1} \times G_{2}\right)$. A quadruple $\left(B_{1}, B_{2}, \delta_{1}, \delta_{2}\right)$ is called SBV for the relation $T$ (see [6] and the references therein), if $\delta$ maps continuously $T$ onto $B_{1} \times B_{2}$ and the restriction of $\delta_{1}$ to $\operatorname{Ker} T$ is a one-to-one mapping of Ker $T$ onto $B_{1}$. We define the operator $\Phi_{\delta}: B_{1} \rightarrow B_{2}$ and the relation $T_{0}$ by the equalities $\Phi_{\delta}=\delta_{2}\left(\left.\delta_{1}\right|_{\text {Ker T }}\right)^{-1}, T_{0}=\operatorname{ker} \delta$. We note that operator $\Phi_{\delta}$ is bounded. We shall say that the relation $T_{0}$ is the minimal relation generated by SBV. It follows from the definition of SBV that there exists a one-to-one correspondence between relations $\widehat{T}$ with the property $T_{0} \subset$ $\subset \widehat{T} \subset T$ and relations $\theta \subset B_{1} \times B_{2}$ and this correspondence is determined by the equality $\delta \widehat{T}=\theta$. In this case we denote $\widehat{T}=T_{\theta}$.

Let $S$ be a linear relation $S \subset B_{1}^{\prime} \times B_{2}^{\prime}$, where $B_{1}^{\prime}, B_{2}^{\prime}$ are Banach spaces. The following conditions are borrowed from [1], [2]: 1) $S$ is closed; $2) \operatorname{ker} S=\{0\}$; 3) $\operatorname{dim} \operatorname{ker} S<\infty ; 4)$ the relation $S$ is well-defined (i.e., $S$ is invertible and the range $\mathcal{R}(S)$ is closed); 5) $\overline{\mathcal{R}(S)}=\mathcal{R}(S) ; 6) \mathcal{R}(S)$ is a closed subspace in $B_{2}^{\prime}$ of the finite codimension; 7) $\mathcal{R}(S)=B_{2}^{\prime}$; 8) $S$ is continuously invertible. Following [1, 2], we shall say that the relation $S$ is in the state $k$ if it satisfies condition $k$ ). The relation $S$ is called Fredholm if it satisfies conditions 3), 6). 
Theorem 4. Let $\mathcal{R}(T)=\mathbf{B}_{2}$. The relation $T_{\theta}$ is in the state $k(1 \leqslant k \leqslant 8)$ if and only if the same is true for the relation $\theta-\Phi_{\delta}$.

The proof is implied by the following lemma established in 6.

Lemma 7 . The relation $T_{\theta}$ is closed if and only if the relation $\theta$ is closed. Suppose $\mathcal{R}(T)=\mathbf{B}_{2}$. The following statements hold true:

1) the range of $\mathcal{R}\left(T_{\theta}\right)$ is closed if and only if the range of $\mathcal{R}\left(\theta-\Phi_{\delta}\right)$ is closed;

2) $\operatorname{dim} \mathbf{B}_{2} / \overline{\mathcal{R}\left(T_{\theta}\right)}=\operatorname{dim} B_{2} / \overline{\mathcal{R}\left(\theta-\Phi_{\delta}\right)}$;

3) $\operatorname{dim} \operatorname{ker} T_{\theta}=\operatorname{dim} \operatorname{ker}\left(\theta-\Phi_{\delta}\right)$.

Let us construct examples of spaces of boundary values for the maximal relation $L$.

Example 1. Let $\widehat{Q}_{0}$ be a set of elements $x \in H$ such that the function $s \rightarrow U^{*}\left(b_{0}, s\right) x$ is identified with zero in $\mathfrak{H}$. We put $\widehat{Q}=H \ominus \widehat{Q}_{0}$. On the linear manifold $\widehat{Q}$, we introduce the norm

$$
\|\widehat{c}\|_{-}=\left\|U\left(b_{0}, \cdot\right)\right\|_{\mathbf{m}}=\left(\int_{a}^{b_{0}}\left(d \mathbf{m}(\xi) U^{*}\left(b_{0}, \xi\right) \widehat{c}, U^{*}\left(b_{0}, \xi\right) \widehat{c}\right)\right)^{1 / 2},
$$

where $\widehat{c} \in \widehat{Q}$. Using (4), (6), we obtain

$$
\|\widehat{c}\|_{-}=\left(\int_{a}^{b_{0}}\left(\Psi_{\mathbf{m}}(s) U^{*}\left(b_{0}, \xi\right) \widehat{c}, U^{*}\left(b_{0}, \xi\right) \widehat{c}\right) d \rho\right)^{1 / 2} \leqslant \gamma\|\widehat{c}\|, \quad \widehat{c} \in Q .
$$

By $\widehat{Q}_{-}$denote the completion $\widehat{Q}$ of with respect to the norm $(40)$. It follows from (41) that the space $\widehat{Q}_{-}$can be treated as a space with a negative norm with respect to $Q$ [4, Ch. 1]. By $\widehat{Q}_{+}$we denote the associated space with a positive norm. The definition of spaces with positive and negative norms imply that $\widehat{Q}_{+} \subset Q$.

Suppose that a sequence $\left\{\widehat{c}_{n}\right\}\left(\widehat{c}_{n} \in \widehat{Q}\right)$ converges in $\widehat{Q}_{-}$to $\widehat{c}_{0} \in \widehat{Q}_{-}$. Then a sequence $\left\{U^{*}\left(b_{0}, \cdot\right) \widehat{c}_{n}\right\}$ is fundamental in $\mathfrak{H}$. It follows that this sequence converges to some element in $\mathfrak{H}$. By $U^{*}\left(b_{0}, \cdot\right) \widehat{c}_{0}$ we denote this element and $\mathcal{V}$ stands for the operator $\widehat{c} \rightarrow U^{*}\left(b_{0}, \cdot\right) \widehat{c}$, where $\widehat{c} \in \widehat{Q}$. . The operator $\mathcal{V}: \widehat{Q}_{-} \rightarrow \mathfrak{H}$ is continuous, one-to-one, and its the range of values is closed. Thus adjoint operator $\mathcal{V}^{*}$ maps continuously $\mathfrak{H}$ onto $\widehat{Q}_{+}$. We shall find the form of the operator $\mathcal{V}^{*}$. For all $x \in \widehat{Q}, f \in \mathfrak{H}$, we have 


$$
\begin{aligned}
(f, \mathcal{V} x)_{\mathbf{m}}=\int_{a}^{b_{0}}\left(d \mathbf{m}(s) f(s), U^{*}\left(b_{0}, s\right) x\right)= \\
=\int_{a}^{b_{0}}\left(U\left(b_{0}, s\right) d \mathbf{m}(s) f(s), x\right)=\left(\mathcal{V}^{*} f, x\right) .
\end{aligned}
$$

Hence, taking into account that $Q$ is densely embedded in $Q_{-}$, we obtain

$$
\mathcal{V}^{*} f=\int_{a}^{b_{0}} U\left(b_{0}, s\right) d \mathbf{m}(s) f(s) \text {. }
$$

Thus the following statement is obtained.

Lemma 8. The operator $\mathcal{V}^{*}$ maps continuously $\mathfrak{H}$ onto $\widehat{Q}_{+}$and is given by 42 .

In accordance with Theorem 3 , a pair $\{\tilde{y}, \tilde{f}\} \in \mathfrak{H} \times \mathfrak{H}$ belongs to the maximal relation $L$ if and only if there exists a pair $\{y, f\}$ such that the pairs $\{\tilde{y}, \tilde{f}\},\{y, f\}$ are identified in $\mathfrak{H} \times \mathfrak{H}$ with each other and equality (39) holds for $\{y, f\}$.

With each pair $\{y, f\}$ represented by $(39)$ we associate a pair of boundary values

$$
\mathrm{Y}=\delta_{1}\{y, f\}=c \in Q_{-}, \quad \mathrm{Y}^{\prime}=\delta_{2}\{y, f\}=\int_{a}^{b_{0}} U\left(b_{0}, s\right) d \mathbf{m}(s) f(s)=\mathcal{V}^{*} f \in \widehat{Q}_{+} .
$$

It follows from $(39)$ that if pairs $\{y, f\},\{\tilde{y}, \tilde{f}\} \in L$ are identified in $\mathfrak{H} \times \mathfrak{H}$, then their boundary values coincide.

We note that if $c \in Q$ (i.e., $\{y, f\} \in L^{\prime}$ ), then

$$
\mathrm{Y}=y(a), \quad \mathrm{Y}^{\prime}=y\left(b_{0}\right)-U\left(b_{0}, a\right) y(a) .
$$

We put $\delta\{y, f\}=\left\{\mathrm{Y}, \mathrm{Y}^{\prime}\right\}$. It follows from Theorem 3, Lemma 8 , Corollary 2 that a quadruple $\left(Q_{-}, \widehat{Q}_{+}, \delta_{1}, \delta_{2}\right)$ is a SBV for relation $L$; at that, $\Phi_{\delta}=0$. We set $\operatorname{ker} \delta=L_{0}$ and call $L_{0}$ the minimal relation generated by equation (3) and boundary values (43). The relation $L_{0}$ is closed. By (43), 44), it follows that the relation $L_{0}$ is a restriction of $L$ to a set of pairs $\{y, f\} \in L$ such that $y(a)=y\left(b_{0}\right)=0$. As above, $L_{\theta}$ is a linear 
relation such that $L_{0} \subset L_{\theta} \subset L$ and $\delta L_{\theta}=\theta \subset Q_{-} \times \widehat{Q}_{+}$. Theorem 4 implies the following statement.

Theorem 5. The relation $L_{\theta}$ is in the state $k$ if and only if the same is true for the relation $\theta$.

Example 2. Let the space $H$ be finite-dimensional, $H=\mathbb{C}^{n}$. In this case the spaces $Q_{0}, Q=H \ominus Q_{0}$ are finite-dimensional and $Q_{-}=Q$. With each pair $\{y, f\}$ represented by 39 we associate a pair of boundary values

$$
\mathrm{Y}=\delta_{1}\{y, f\}=y(a)=c, \quad \mathrm{Y}^{\prime}=\delta_{2}\{y, f\}=\int_{a}^{b_{0}} d \mathbf{m}(s) y(s) .
$$

By $Q_{2}$ denote the range of the operator $\delta_{2}, Q_{2}=\mathcal{R}\left(\delta_{2}\right) \subset H$. It follows from Theorem 3 . Corollary 2 that a quadruple $\left(Q, Q_{2}, \delta_{1}, \delta_{2}\right)$ is a SBV for the relation $L$; at that, $\Phi_{\delta} c=\int_{a}^{b_{0}} d \mathbf{m}(s) U(s, a) c, c \in Q$. The minimal relation $L_{0}$ is a restriction of $L$ to a set of pairs $\{y, f\} \in L$ such that $y(a)=\delta_{2}\{y, f\}=0$. Theorem 4 implies the following statement.

Theorem 6. The relation $L_{\theta}$ is in the state $k$ if and only if the same is true for the relation $\theta-\Phi_{\delta}$.

Example 3. Let the space $H$ be finite-dimensional, $H=\mathbb{C}^{n}$. Suppose that $\mathbf{m}=\mu E$ is the usual Lebesque measure on $[a, b]$, i.e., $\mu([\alpha, \beta))=\beta-\alpha$, $a \leqslant \alpha<\beta \leqslant b$ (as above, we let $\mu(\Delta)=0$ for each Borel set $\Delta$ such that $\left.\Delta \subset\left(b, b_{0}\right]\right)$. In this case the maximal relation $L$ is an operator. We assume that $\mathbf{p}(\{a\})=\mathbf{p}(\{b\})=0$. Then $Q=Q_{-}=\widehat{Q}=\widehat{Q}_{-}=H$ and $y\left(b_{0}\right)=y(b)$. With each pair $\{y, f\} \in L$ we associate a pair of boundary values

$$
\mathrm{Y}=\delta_{1}\{y, f\}=y(a) \in H, \quad \mathrm{Y}^{\prime}=\delta_{2}\{y, f\}=\{y(\zeta), y(b)\} \in H \times H,
$$

where $a<\zeta<b$. The space $H \times H$ is the range of the operator $\delta_{2}$. It follows from Theorem 3 that a quadruple $\left(H, H \times H, \delta_{1}, \delta_{2}\right)$ is a SBV for the operator $L$; at that, $\Phi_{\delta} c=\{U(\zeta, a) c, U(b, a) c\}$. The minimal operator $L_{0}$ is a restriction of $L$ to a set of $y \in \mathcal{D}(L)$ such that $y(a)=y(\zeta)=y(b)=0$. Theorem 4 implies a statement similar to Theorem 6 .

Example 4. Under the condition of Example 3, we define the measure p by the equality

$$
\mathbf{p}(\Delta)=\int_{\Delta} B(s) d \mu(s)+\mathbf{p}_{\zeta}(\Delta)
$$


for all Borel sets $\Delta \subset[a, b]$ (as above, $\mathbf{p}(\Delta)=0$ if $\Delta \subset\left(b, b_{0}\right]$ ). Here $s \rightarrow B(s)$ is a measurable function whose values are linear operators in $H$, at that, the function $s \rightarrow\|B(s)\|$ belongs to $L_{1}(a, b) ; a<\zeta<b$; $\mathbf{p}_{\zeta}(\Delta)=0$ for each Borel set $\Delta$ such that $\Delta \cap\{\zeta\}=\varnothing$ and $\mathbf{p}_{\zeta}(\{\zeta\})=A$; $A$ is a bounded operator in $H$. Then equation (3) takes the form

$$
y(t)=c+\int_{a}^{t} B(s) y(s) d \mu(s)+\int_{a}^{t}\left(d \mathbf{p}_{\zeta}\right) y(s)+\int_{a}^{t} f(s) d \mu(s), \quad c \in H .
$$

Therefore, $y^{\prime}(t)=B(t) y(t)+f(t)$ if $t \neq \zeta$ and $y(\zeta+0)=y(\zeta)+A y(\zeta)$. So, $y$ is a solution of the differential equation with an impulse action (see [11). The presence in boundary conditions of a linear relation $\theta$ allows us to consider equations with multi-valued impulse actions.

\section{References}

[1] Baskakov A. G. Spectral Analysis of Differential Operators with Unbounded Operator-valued Coefficients, Difference Relations and Semigroups of Difference Relations. Izv. Math., 2009, vol. 73, no 2, pp. 215-278. DOI: 10.1070/IM2009v073n02ABEH002445. [Russian edition: Izv. RAN. Ser. Matem., 2009, vol. 73, no 2, pp. 3-68. DOI: 10.4213/im2643.]

[2] Baskakov A. G. Analysis of Linear Differential Equations by Methods of the Spectral Theory of Difference Operators and Linear Relations. Russian Math. Surveys, 2013, vol. 68, no 1, pp. 69-116. DOI: 10.1070/RM2013v068n01ABEH004822. [Russian edition: Uspekhi Mat. Nauk, 2013, vol. 68, no 1, pp. 77-128. DOI: 10.4213/rm9505.]

[3] Behrndt Jussi, Hassi Seppo, Snoo Henk, Wietsma Rudi. Squareintegrable Solutions and Weil Functions for Singular Canonical Systems. Math. Nachr., 2011, vol. 284, no 11-12, pp. 1334-1384. DOI: 10.1002/mana.201000017.

[4] Berezanski Yu. M. Expansions in Eigenfunctions of Selfadjoint Operators. Amer. Math. Soc., Providence, RI, 1968. [Russian edition: Naukova Dumka, Kiev, 1965.]

[5] Bruk V. M. Linear Relations in a Space of Vector Functions. Math. Notes, 1978, vol 24, no 4, pp. 767-773. DOI: 10.1007/BF01099164 [Russian edition: Mat. Zametki, 1978, vol. 24, no. 4, pp. 499-511.]

[6] Bruk V. M. On Linear Relations Generated by Nonnegative Operator Function and Degenerate Elliptic Differential-Operator Expression. J. Math. Phys., Anal., Geom., 2009, vol. 5, no 2, pp. 123-144. 
[7] Bruk V. M. On the Characteristic Operator of an Integral Equation with a Nevanlinna Measure in the Infinite-Dimensional Case. J. Math. Phys., Anal., Geom., 2014, vol. 10, no 2, pp. 163-188. DOI: 10.15407/mag10.02.

[8] Cross R. Multivalued Linear Operators. Monogr. Textbooks Pure Appl. Math., 213, Dekker, New York, 1998.

[9] Orcutt B. C. Canonical Differential Equations. Dissertation, University of Virginia, 1969.

[10] Rofe-Beketov F. S. Selfadjoint Extensions of Differential Operators in a Space of Vector functions. Soviet. Math. Dokl., 1969, vol. 10, no 1, pp. 188192. [Russian edition: Dokl. Akad. Nauk SSSR, 1969, vol. 184, no 5, pp. 1034-1037.]

[11] Samoilenko A. M., Perestyuk N. A., Impulsive Differential Equations. World Scientific Series on Nonlinear Science, Ser. A, vol. 14, Singapore, 1995. [Russian edition: Vischa Schkola, Kiev, 1987.]

Received April 21, 2017.

In revised form, June 15, $201 \%$.

Accepted June 19, $201 \%$.

Published online June 22, $201 \%$.

Saratov State Technical University

77, Politehnicheskaja str., Saratov 410054, Russia

E-mail: vladislavbruk@mail.ru 